\title{
Pathology and operative strategy
}

\section{A Reddi}

\section{What if a preoperative diagnosis is unavailable?}

A s a general principle, in elective surgery, it is considered advisable to establish a pathological diagnosis (by cytology or histology, and occasionally by microbiological means) before the removal of a lesion, with or without the associated organ or part thereof. The rationale for this approach is obvious: to rule out the possibility of an alternative form of treatment - that is, a more conservative one-that will obviate the need for surgical resection. Take-for example, endobronchial tuberculosis, which may on clinical, radiographical, and endoscopical grounds be indistinguishable from bronchial carcinoma. In this instance, to remove a lung or lobe without histological confirmation would clearly be catastrophic. However, it is not always possible to obtain tissue for preoperative diagnosis. Inaccessible organs/lesions or the invasive nature of the diagnostic manoeuvre may make the procedure hazardous, with a statistically significant morbidity and, occasionally, even mortality. Under these circumstances, the clinician may have to resort to an amalgam of clinical, radiographical, and ancillary biochemical investigations for a diagnosis. Where the treatment is deemed surgical, even without a definitive pathological diagnosis, the surgeon is justified in resection of the lesion (with or without the associated viscous), based on a provisional diagnosis. Tissue diagnosis by frozen section is thought advisable, although less reliable than a paraffin wax embedded section.

A wide variety of medical personnel, influenced by local practices and available expertise, undertake minor diagnostic procedures (usually without the need for a general anaesthetic). Where fee for service structures exist, the incentive for financial remuneration may determine the investigator. This "generalist" approach to diagnosis is acceptable where the manoeuvre is relatively simple and innocuous and the diagnostic yield is high irrespective of the technical expertise required. However, where there is a risk of serious complications, and the detection and management thereof mandates expert vigilance and treatment by skilled practitioners, then discretion and prudence should prevail. Take the example of fine needle aspiration cytology of a lung lesion, which may be complicated by a pneumothorax or haemoptysis, and very rarely requires operative intervention. Should the procedure be undertaken by a pulmonologist, radiologist, pathologist, or thoracic surgeon? Undoubtedly, each specialty contributes its own skill and expertise in determining the nature of the lesion. Nonetheless, what dictates who should perform the invasive investigation? Technical skill, a high diagnostic yield, and a negligible complication rate is the prerequisite ideal. With experience, these may be easily achieved. However, the detection and treatment of a complication has immense practical value in determining who undertakes the invasive procedure, in this particular instance, where technical expertise is equally shared. Furthermore, due consideration must be given to the specialty that will ultimately determine the definitive treatment, and possibly undertake it. Logically, this specialty should perform the diagnostic procedure to expedite treatment and avoid the inevitable delay inherent in interdisciplinary referrals.

"Removal of a lesion operatively
shown to be otherwise treatable
by pharmacological or more
conservative means exposes the
surgeon to medicolegal
interrogation"

Occasionally, despite exhaustive investigative modalities, including frozen section, a pathological diagnosis is not reached, and the surgeon is forced to make an operative decision, based on the macroscopic appearances of the morbid anatomy. A thrombus in the right atrium may be confused with a right atrial myxoma. Logistically, frozen section diagnosis may increase the duration of the ischaemic time, and thus adversely affect patient outcome, making it impractical. It is then that the clinical assessment of the surgeon is called to judgement-is the action of surgical removal reasonable given the findings? One must now delve into the nebulous sphere of subjective assessment to decide what is "reasonable". Factor in the incentive for financial remuneration for service rendered and the situation becomes even more complex. Internet, television, and other large scale information systems have made the affluent patient more aware of the advances in medical knowledge and technology, often encouraging the patient to demand or question the need for specific medical procedures. The potential for litigation has serious implications for surgical decision making. Removal of a lesion operatively shown to be otherwise treatable by pharmacological or more conservative means exposes the surgeon to medicolegal interrogation. One may consider judging the individual case by its surgical outcome; if the postoperative course is uneventful, with no harm done, and the patient is spared the prolonged anxiety and doubt caused by the absence of a diagnosis, then the surgical action may be justified, especially when the intention to cure is proved ultimately to be the abiding motive in performing the procedure, despite, on retrospective analysis, the availability of an alternative conservative form of treatment.

It is well known and generally accepted that for accurate pathological analysis, an adequate and representative tissue sample is necessary; a wedge biopsy taken from the periphery of a lesion may be more useful than a core from the centre of a necrotic tumour; an early morning (cough) specimen of sputum may have a greater diagnostic yield than a later one; a midstream urine sample is less likely to contain contaminants that will interfere with the diagnosis than a random specimen. A diagnostic dilemma arises when the lesion contains dual pathology or when the lesion is known to be associated with a less sinister disease-for example, granulomatous mediastinal lymphadenitis may be associated with bronchial carcinoma. Biopsy material obtained at mediastinoscopy may erroneously ascribe a lesion elsewhere in the thorax-for example, the lung-as tuberculous or sarcoid when it is actually neoplastic with associated mediastinal lymphadenopathy. Similarly, satellite nodules at the periphery of a bronchial carcinoma may yield a diagnosis of an inflammatory lesion when the primary pathology is malignant.

We now arrive at the crucial question-how accurate is a histological diagnosis? The obvious and immediate response would be to state categorically: as accurate as the sample represented. The question should therefore be rephrased. What conclusion regarding a diagnosis can be drawn from the histology? In most cases, histology is the final arbiter in diagnosis. Should the clinician place absolute trust in this most definitive form of diagnosis? As with all other areas of medicine, a diagnosis is and should always be an amalgam of a coherent and relevant history, a thorough clinical examination correlated (where necessary) with appropriate ancillary investigations, and a relevant 
tissue analysis where possible. An accurate diagnosis, in its broadest sense, is the single most important prerequisite for appropriate and meaningful treatment. If the clinical features are at odds with the investigations or vice versa, then a complete reappraisal of all findings and results is absolutely essential if the patient is to derive the benefit of an accurate diagnosis, and hence appropriate treatment.

The artificial sequestration of pathologists remote from the clinical arena deserves comment, if not re-evaluation of the present modus operandi of the discipline. Pathology textbooks are veritable storehouses of clinical information, in addition to the obligatory dissertations on morbid anatomy. Forums for interaction between clinicians and pathologists are sparse and unfortunately pursue divergent or parallel pathways. This relationship is a keystone and forms the basis of clinical practice. Frontiers have been forged in molecular pathology, terra incognita, for present day medical practitioners. For the pathologist, a clinicopathological correlation is complete

only with the postmortem examination, when precise information on the history, clinical examination, and ancillary investigations is available. In the clinical sphere, the pathologist should be similarly involved and informed to make the evaluation of specimens more meaningful.

\begin{abstract}
"An accurate diagnosis, in its broadest sense, is the single most important prerequisite for appropriate and meaningful treatment"
\end{abstract}

A surgeon's primary intention after diagnostic manoeuvres, in a patient deemed operable, is to restore normal or near normal anatomy. Where this is not possible, then at least a functional one is acceptable. Histological diagnosis or special investigations are not always crucial in planning operative strategy; pectus excavatum requires visual appraisal only, whereas an aortic dissection of the ascending aorta with severe aortic regurgitation requires a gamut of special investigations and the expertise of ancillary specialists. Treatment is based on morbid anatomy determined by radiological means only. In this instance, histology is an accessory after the fact of operative treatment. In other words, where the pathology has, primarily, a mechanical effect with disturbance of physiological function, then the surgical objective would be to treat the effect by removal of the pathology or, where not possible, to circumvent the effect using well known surgical techniques. This last approach is usually palliative.

For operative treatment, a pathological diagnosis when necessary is preferred, by a benign and expeditious route, if possible.

\section{Authors' affiliations}

Correspondence to: Mr A Reddi, Department of Cardiothoracic Surgery, Wentworth Hospital, Nelson R Mandela School of Medicine, University of Natal, Durban, South Africa welman@nu.ac.za

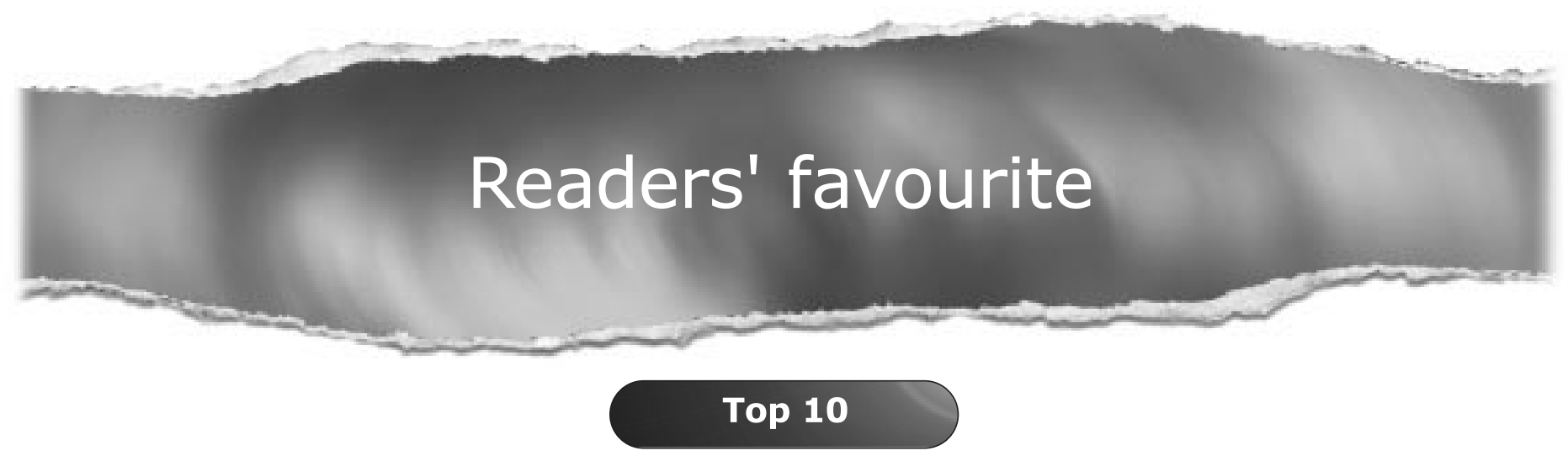

Click on the "Top 10" button on the homepage to see which are the best read articles each month

www.jclinpath.com 